\title{
A Novel Approach in Long Range Extended Remote I/O using FAMPLC Technique
}

\author{
A. Nazem ${ }^{1 *}$ and M. R. Arshad ${ }^{2}$ \\ 1,2Underwater Robotics Research Group (URRG), School of Electrical and Electronic Engineering, Universiti Sains \\ Malaysia, NibongTebal, Pulau Pinang, Malaysia; alireza_nazem@ftzlogic.com, rizal@eng.usm.my
}

\begin{abstract}
Background/Objectives: To introduce a novel achievement in distant access to an I/O port using Frequency and Amplitude Modulations in Power Line Communication (FAMPLC) technique.

Methods/Statistical Analysis: FAMPLC is a platform for large signal communication over a power line. A Frequency Modulated Power Supply (FMPS) as a power and data gateway to supply and communicate with the remote (slave) module is developed, which is called the Master (or server) module. The transmission procedure from the slave node to the master unit is performed by use of an active resistance as a dummy load to overlay pulses onto the consumer's current.

Results: The experimental outcomes of a full - duplex 16 bits (8×8) extended remote I/O module without the requirement of any localized power supply and with the distance range enhancement, up to twelve kilometers are presented throughout this paper.

Conclusion/Application: A full-duplex development of a remote I/O module is presented. With this development, an unshielded pair of wires may be used and longer distance range can be achieved.
\end{abstract}

Keywords: FAMPLC, FMPS, Long Range Extended Remote I/O, Active Resistance, Dummy Load.

\section{Introduction}

One of the most attractive achievements of the novel technique of FAMPLC belongs to the experimental development of an extended remote I/O. Input and Output connections and configurations are inherent blocks of a control system. For many particular reasons such as in underwater applications or more specifically in accordance with the safety regulations in petroleum industry, an I/O block may be required to be detached from the main controller. The most common method using the Supervisory Control and Data Acquisition (SCADA) system [1] is today's practice in conjunction with Programmable Logic Controllers (PLC) [2]. However, one important mater about these peripherals is the requirement of a localized power supply. By using FAMPLC technique, this issue will be addressed besides

\footnotetext{
* Corresponding author:
}

A. Nazem (alireza_nazem@fltzlogic.com) the benefit of reaching longer distance ranges. FAMPLC is introduced as a non-superimposing method in which the power-line is utilized as a communication platform using its parametric values such as Voltage, Current, frequency and duty cycle [3]. A dedicated DC to AC inverter is designed as the power and data gateway for this purpose, which is called the Master (or server) module [4]. This technique is a standalone embedded design containing a square wave Frequency Modulated Power supply (FMPS) and Thevenin Equivalent Converter (TEC) circuitries. The receiver node as a communicating point is to extract the means of commanding data by analysing the power pulse widths. The transmission procedure from the receiver node to the master module is then performed using an active resistance as a dummy load. A successful development of this technique in a Remotely Operated Vehicle (ROV) application is discussed in [5]. 
According to the fundamental concept of this technique which is exhaustively discussed in [3], as long as the slave node's circuitry is supplied with sufficient power to drive the active switches, the transmission length can be increased. As discussed in [6], in fact, if voltage stability exists, the power components of the system will be controllable at all times. Since in an extended I/O device the slave module is not designed as a power gateway, and also port switches are chosen from active components with isolated gates (Figure 1), the power drain by the slave node is minimal and just limited to its circuitry. Therefore, very small amount of drop across the line will occur and thus, very long communication distance over the power line is expected. In Figure 2 the developed model for an " $8 \times 8$ " $\mathrm{I} / \mathrm{O}$ connection is drawn.

In the herewith presented development, twelve kilometres distance between Master and slave units is successfully achieved and it is yet to be increased performing further improvements by inclining toward Nano level consumption. However, practically by increasing the DC voltage level in the inverter block, the length can still be increased proportionally, compensating the voltage drop due to the copper loss.

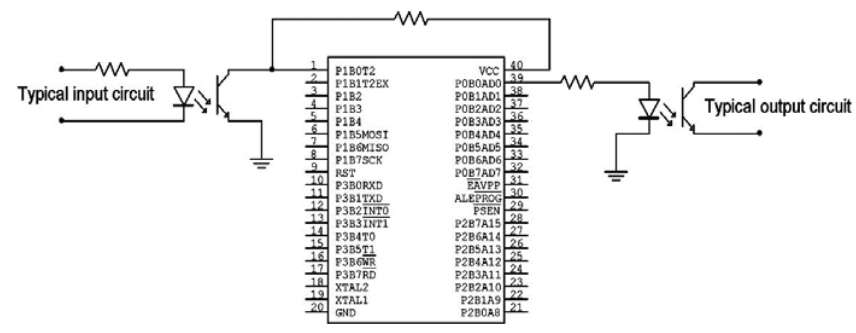

Figure 1. I/O Hardware architecture.

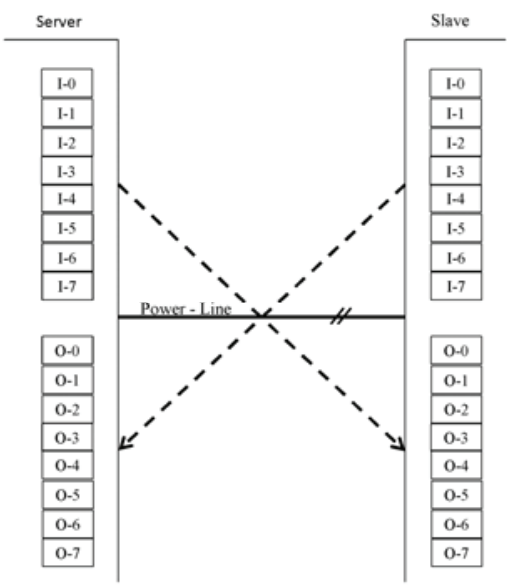

Figure 2. The concept of the extended remote I/O.

\section{Approach and Methods}

With respect to the conceptual aspects of FAMPLC technique, a counting based process is performed in both modules. The Voltage (power) pulse widths and the current overlaid pulse widths are to carry the means of port conditions. In an 8 bit configuration, 256 conditions are available in a port. In other words, the individual bit values are considered in a byte form. For example, in an occasion where inputs: I0 to I7 are " $1,1,0,1,0,0,1,1$ " respectively, the value of the port is equal to 203 in decimal mode and CB in hexadecimal. Let's consider the frequency $F_{x}$ and the duty cycle $d_{x}$ as the communication elements. $d_{x}$ in this research is considered within the range of $18-82 \%\left(82 \% \geq d_{x}>18 \%\right)$. Thus, the steps for $d_{x}$ variations are $0.25 \%$ for every sequential condition. As an example, Table 1 tabulates $d_{x}$ for some selected port values.

The selection of the communication frequency $F_{x^{\prime}}$ requires extra challenge figuring out the characteristics of the transmission line. As discussed in [7], pulse edge distortion due to the dynamic effect of the cable, enforces for lower frequency consideration, while counting speed will be affected. Despite existing wire - based transmissions, unshielded cable is more suitable in FAM technique because of its large signal concept. In case of using an unshielded cable both the bulk and inner capacitive effects will be minimal and higher frequencies can be considered. In addition, the cost and effort of installing an unshielded cable or simply a pair of wires are considerably lesser than the other types. In this research, a separate wires in pair are used as FAM transmission line. Therefore, the values of the cable dynamic parameters using AWG 16 wire, are minimized as in Figure 3. Notable that, the given values are measured in 1000 meters of the wires in a pair.

Count losses due to damped edges are measured in a calibration sequence ahead of every pulse train. If the

Table 1. Sample of $d_{x}$ for some selected port values in binary and decimal modes.

\begin{tabular}{lcc}
\hline I7 - I0 & Decimal value & $d_{x}(\%)$ \\
\hline 00000000 & 0 & 18.25 \\
00000001 & 1 & 18.5 \\
00000010 & 2 & 18.75 \\
00001000 & 8 & 20.25 \\
10000000 & 128 & 50.25 \\
11110000 & 240 & 78.25 \\
11111111 & 255 & 82 \\
\hline
\end{tabular}




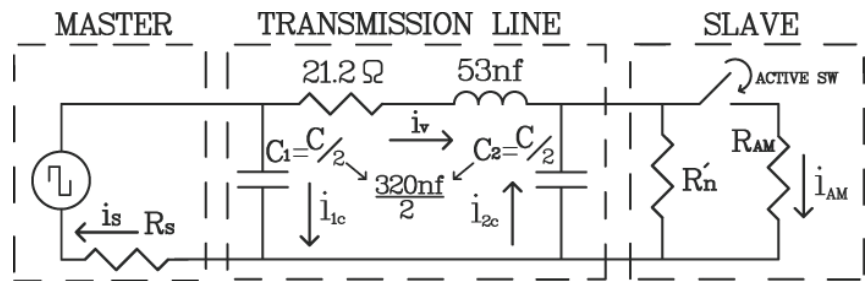

Figure 3. An equivalent model for a wire based transmision line.

Table 2. Example of $\gamma$ and $g$ protective distributed counts for an errorless communication achievement

\begin{tabular}{lcr}
\hline$d_{x}(\%)$ & $C_{p}\left(d_{x}\right)+\gamma \geq C_{p}\left(d_{x}\right) \geq C_{p}\left(d_{x}\right)-\gamma$ & \multicolumn{1}{c}{$g_{c}$} \\
\hline 18.25 & $196-216$ & $217-226$ \\
18.5 & $237-247$ & $248-257$ \\
82 & $8101-8121$ & $8122-8131$ \\
\hline
\end{tabular}

transmission line is fixed and the length and characteristics are constant, calibration sequence may be done just in the initialization routine. On the other hand, instead of a single value, a sequential range of numbers as propagative factor $(\gamma)$ are assigned to each $d_{x}$ counted value. Furthermore, to prevent from overlapping errors, additional sequential numbers $\left(g_{c}\right)$ as don't care values are assigned as the gap between meaningful values. An example of selected ranges are given in Table 2.

Since informative data are carried over separate layers, instant transmission from each party may occur independent from another. Therefore, a full-duplex port scanning process is established.

\section{Result and Discussion}

The DC level at the inverter block is set to $36 \mathrm{~V}$. The slave node consumption varies from $23 \mathrm{~mA}$ to $110 \mathrm{~mA}$ respective to the activated I/O ports. The study of various loading conditions shows that the Total Harmonic Distortion (THD) [8] proportionally increases with the total consumption at the slave node, however compensable by increase in DC level.

Considering the given values in $\Pi$ model of Figure 2, the distorted voltage pulse edge in 12000 meters will be as shown in Figure 4 in comparison with the source pulse.

On the other hand, the active resistance value is set to overlay current pulses at $52.5 \mathrm{~mA}$. The current pulse edge distortion resultin 12000 meters of transmission length is shown in Figure 5 also in comparison with the source pulse.

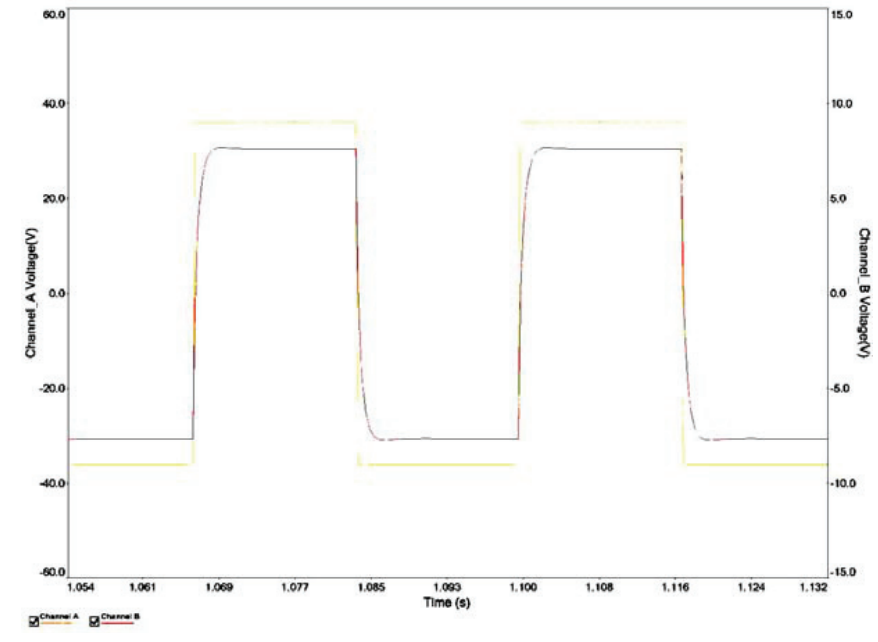

Figure 4. The large signal FMPS output pulse.

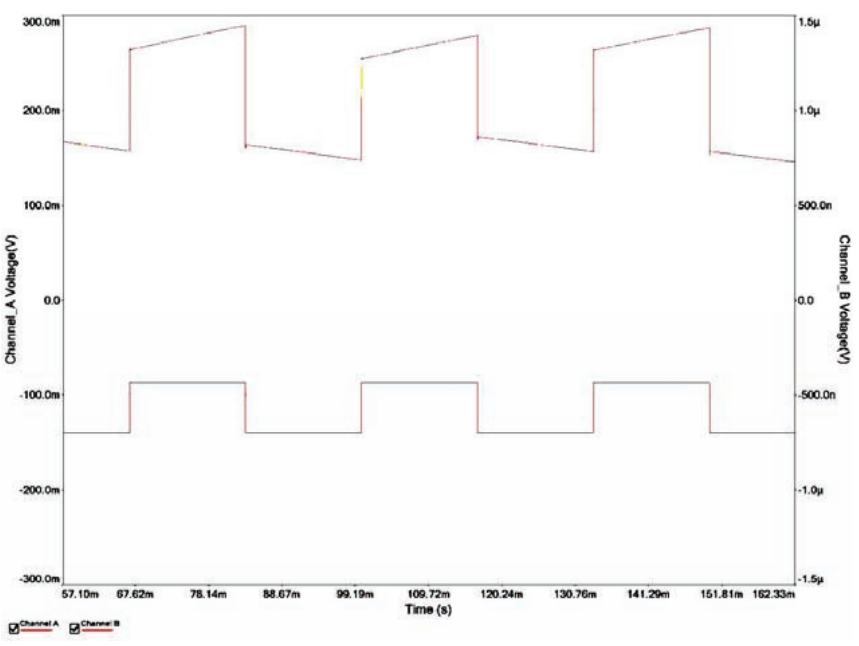

Figure 5. Dummy load activity in the slave module.

Results of both transmissions shows minor interference to the source pulse. However, the supplying voltage (power) results in more damping effects whereby the rising edge delays the counting process. Due to this reason the calibration sequence should be performed in initialization stage.

\subsection{Calibration}

A calibration pulse with a constant value is generated at both master's and slave's initialization sequences. This is meant for internal comparison to evaluate the amount of pulse width loss due to any filtering effect. In this research a definite voltage pulse with parametric values of $F_{c a l}=50 H_{z}$ and $d_{c a l}=50 \%$ are defined in initialization rou- 
tine of both master and slave modules. These values result in $T_{c a l}=10 m s$ which is called the Mark of the pulse. Each party is to transmit this value to another in calibration stage. At another end, the device is taught to compare the received pulse width with the predefined calibration value in its lookup table. Further analysis show that the embedded counter value of the slave node has reached to 4897 (at $F_{c}=500 \mathrm{KHz}$ the counter clock) within the interval of the pulse width. While the predefined counter value of $C_{p}=5000$ had been initially taught to the system, therefore, $C_{c a l}=103$ is assigned as the lost value and will be added to the consequential informative voltage pulses.

\subsection{Communication Frequency}

Due to the establishment of a reliable low noise transmission platform by considering the abovementioned study, the communication frequency in this research has been set proportional to $C_{c a l}$. Knowing the counter clock frequency at $F_{c}=500 \mathrm{KHz}$ results in $t_{c}=2 \mu \mathrm{s}$. Thus, $t\left(C_{c a l}\right)=206 \mu \mathrm{s}$. In this research the narrowest pulse width is considered equal to $2 t\left(C_{c a l}\right)=412 \mu \mathrm{s}$. The protective gap counts $\left(g_{c}\right)$ and the propagation constant $(\gamma)$ values are also set to 10 count for each. Since according to Table 1 the narrowest pulse width is equal to $d_{x}=18.25 \%$ therefore, Mark $(18.25 \%)=412 \mu \mathrm{s}$. Including $\gamma$ and $g_{c}$ into the calculation yields to the requirements of the 10363 pulses when $d_{x}=100 \%$. Therefore, $T_{x}=20.72 m s$ that yields to $F_{x}=48.24 \mathrm{~Hz}$.

\subsection{Checksum Function}

There is no checksum function in the herewith presented development. However, for the purpose of reliability of the system, every refreshed port value is the result of a calculated average of at least ten sequential pulse trains.

\subsection{Respond Time}

Considering, $F_{x}=48.24 \mathrm{~Hz}$ the discussion in section 3.3 and the required processing time for a mid-range microcontroller [9] at $20 \mathrm{Mhz}$, the respond time for each party will be $400 \mathrm{~ms}>t_{r}>80 \mathrm{~ms}$ depends on the measurements time of the receiving pulse width.

\section{Conclusion}

A novel method in which a 12 kilometres extended remote I/O module over a dedicated line is introduced. The development stages of a 16 bit $(8 \times 8)$ master - slave model is also presented. The 8 bit independent inputs are consid- ered as a byte form at one end. The value of the input byte is transferred to another end using the means of a predefined pulse width. A proper selection of the frequency and the duty cycle of the voltage and current pulses, however arbitrary, will result in higher reliability and longer distance achievements.

\section{Acknowledgement}

Thanks to the Universiti Sains Malaysia for facilitating this research by PRGS 1001/PELECT/8043012 grant scheme and caused motivation to approach the research's outcomes.

\section{References}

1. Mollah M B, and Islam S S (2012). Towards IEEE 802.22 based SCADA system for future distributed system, Proceeding of IEEE/OSAIIAPR Conference on Informatics, Electronics \& Vision, Bangladesh, 1075-1080.

2. Talib I B S, and Azman A A (2012). Design and fabrication of programmable logic controller kit with multiple output module for teaching and learning purposes, Proceeding of $8^{\text {th }}$ IEEE International Colloquium on Signal Processing and its Applications (CSPA), Malaysia, 14-18.

3. Nazem A, and Arshad M R (2012). Real time distant communication using frequency and amplitude combined modulations in single source power line, Proceeding of International Conference on Advances in Electrical and Electronics Engineering, Malaysia, 330-335.

4. Nazem A, and Arshad M R (2012). Frequency modulated power supply used to navigate the Remotely Operated Vehicle (ROV), Proceeding of the $4^{\text {th }}$ International Conference on Intelligent and Advanced Systems, Malaysia, 222-225.

5. Nazem A, and Arshad M R (2012). A dummy active resistance to transmit the navigational parameters of an ROV, Journal of Procedia Engineering, vol 41, 175-181.

6. Kamarposhti M A (2010). Evaluation of static synchronous compensator in order to loading margin study in power system, Indian Journal of Science \& Technology, vol 3(5), 511-514.

7. Irwain J D, and Nelms R M (2011). First-and second-order transient circuits, Basic Engineering Circuit Analysis, $10^{\text {th }}$ Edn., John Wiley \& Sons, Inc. USA, 296-368.

8. Liu Y, Hong $\mathrm{H}$ et al. (2009). Real-time calculation of switching angles minimizing THD for multilevel inverters with step modulation, IEEE Transaction on Industrial Electronics, vol 56(2), 285-293.

9. Microchip (1997). Mid-Range MCU Family Reference Manual, PICmicro ${ }^{\mathrm{TM}}$, USA. 